\title{
ENTROPIC CONDITIONS AND HEDGING
}

\author{
SAMUEL NJOH ${ }^{1}$
}

\begin{abstract}
In many markets, especially in energy markets, electricity markets for instance, the detention of the physical asset is quite difficult. This is also the case for crude oil as treated by Davis (2000). So one can identify a good proxy which is an asset (financial or physical) (one)whose the spot price is significantly correlated with the spot price of the underlying (e.g. electicity or crude oil). Generally, the market could become incomplete. We explicit exact hedging strategies for exponential utilities when the risk premium is bounded. Our result is based upon backward stochastic differential equation (BSDE) and a good choice of admissible strategies which allows us to solve our hedging problem.
\end{abstract}

Mathematics Subject Classification. 90A09, 34A12.

Received February 16, 2006. Revised September 6 and October 12, 2006.

\section{INTRODUCTION}

The issue of the hedge of an European option admits in the situation of incomplete markets many answers by optimization's programs. We aim to study this question and exhibit optimal strategies for exponential utilities. On the subject, the research for the elaboration of an exact solution is still open. We intend to solve the problem when the risk premium is bounded. From an economical view, we consider an agent who sells an option quantified by an $\mathcal{F}_{T}$-measurable contingent claim almost surely finite $H$; with $\mathcal{F}_{T}$ the information available up to the date $T, T$ being the maturity of the option. The agent constitutes a portfolio with an initial wealth and invests in the asset of correlated spot price and in a numéraire. The agent can also decide not to sell. It seems natural to introduce an utility function $U_{\gamma}$ which quantifies the preferences of the investor. Here,

$$
U_{\gamma}(x)=-\exp (-\gamma x)
$$

with $\gamma$ a strictly positive constant. Consequently our agent maximizes its terminal net wealth by the bias of $U_{\gamma}$.

Our presentation of hedging by exponential utility is based upon [4]. We use duality methods and elaborate a good setting for the solving of our optimization problem. First the originality of our work is to introduce several sets of probability and admissible strategies that resume the difficulties and are useful to better overcome them. Hence, we succeed to well define and characterize the optimal hedging strategy. We have tried here to give a complete setting when the risk premium is bounded. To characterize the optimal solution, we use backward stochastic differential equations.

\footnotetext{
Keywords and phrases. Stochastic optimization, martingale representation theorem.

1 Université de Marne-La-Vallée, Cité Descartes, 5, Bld Descartes, Champs-Sur-Marne, 77454 Marne-La-Vallée Cedex 2, France; njoh@math.univ-mlv.fr
} 
By taking the definition of admissible strategies in [4] rather than the one in [8], we give complete proofs of results of [8]. The document is organized as follows. First, the Section 1 will recall some general results about the risk neutral pricing in a general financial model; also we will establish a martingale representation theorem when one changes the probability measure. We also focus on admissible strategies's space and no arbitrage opportunities. Our setting deals with cross hedging, see also [4]. But the cross hedging problem can be viewed as a constraint on hedging portfolio and henceforth enter in the framework regarded in [8]. Our Section 2 is devoted to the characterization of dual problem. We point out the set of martingale measures which allows us to elaborate the dual problem. The Section 3 is concerned by the resolution of the dual problem using backward stochastic differential equations. Indeed, we do verify that the optimal solution is in the set of measure we have previously defined. The Section 4 solves thoroughly the problem of optimal hedging. We look also at the indifference price when the correlation is perfect. In Section 5 we conclude.

\section{Preliminaries}

We consider a financial market with two risky assets and a riskless asset, namely a bond. We make the assumption that one of the risky asset cannot be detained in a financial portfolio. Let $S^{e}$ and $S^{g}$ be the spot prices processes of the risky assets and let $S^{0} \equiv 1$ be the one of the asset without risk. We consider that the physical stock of price $S^{e}$ cannot be exchanged in the market, though it is taken as the underlying of contingent claims.

We recall the definition of a martingale measure:

Definition 1.1. A probability measure $\mathbb{Q}$ is a martingale measure if $\mathbb{Q}$ is equivalent to $\mathbb{P}$ on $\mathcal{F}_{T}$ and the process $S^{g}$ is a local martingale under $\mathbb{Q}$. Let $\mathcal{M}_{e}$ be the set of martingale measures.

\subsection{Duality concept}

Let $V_{T}^{x, \theta}$ be the terminal value of a trading self-financing portfolio process of values $V^{x, \theta}$, with $x$ being the initial wealth of the investor. One of our goal on this work is to prove a duality result on the form

$$
\sup _{\theta \in \mathcal{A}(x)} \mathbb{E}\left[-\exp \left(-\gamma\left(V_{T}^{x, \theta}-H\right)\right)\right]=-\exp \left(\gamma \sup _{\mathbb{Q} \in \mathcal{M}_{e}}\left(\mathbb{E}^{\mathbb{Q}}(H)-x-\frac{1}{\gamma} h(\mathbb{Q} \mid \mathbb{P})\right)\right)
$$

with $h(\mathbb{Q} \mid \mathbb{P})$ denoting the relative entropy of $\mathbb{Q}$ with respect to $\mathbb{P}$ we will precise in the following section. Then the resulting strategy will be optimal for an exponential utility criterion. We notice that the case $H \equiv 0$ recovers a pure investment problem. Beyond the dual problem, we see that one has to minimize the relative entropy minus a penalizing term depending on $H$. The greatest task perhaps will then be to construct the martingale measure $\hat{\mathbb{Q}}$ which allows to get out the optimal trading strategy. Indeed it is necessary to prove that $\hat{\mathbb{Q}}$ is in a subset of $\mathcal{M}_{e}$. We will also give in the Section 2 some conditions on $H$ for the solving of the dual problem in our approach. First of all we recall results obtained.

\subsection{Our results}

Let $B$ be a random variable almost surely finite. Let $\mathcal{A}_{b}(x)$ and $\mathcal{A}(x)$ be two spaces of admissible strategies we define latter in the section. We set

$$
\begin{gathered}
v(x, B):=\sup _{\theta \in \mathcal{A}(x)} \mathbb{E}\left[-\mathrm{e}^{-\gamma\left(V_{T}^{x, \theta}-B\right)}\right] \\
V(x, B)=\frac{1}{\gamma} \ln [-v(x, B)] \\
\mathcal{M}_{\theta}^{\prime}:=\left\{\mathbb{Q} \ll \mathbb{P} \mid V^{x, \theta} \mathbb{Q} \text {-integrable and } h(\mathbb{Q} \mid \mathbb{P})<+\infty\right\} .
\end{gathered}
$$

Then we establish that: 
- If $\mathbb{E}\left(B \mathrm{e}^{B}\right)<\infty$ and if $\theta \in \mathcal{A}_{b}(x)$, then

$$
\frac{1}{\gamma} \ln \mathbb{E}\left[\exp \left(-\gamma\left(V_{T}^{x, \theta}-B\right)\right)\right]=\sup _{\mathbb{Q} \in \mathcal{M}_{\theta}^{\prime}}\left\{\mathbb{E}^{\mathbb{Q}}\left[-V_{T}^{x, \theta}+B\right]-\frac{1}{\gamma} h(\mathbb{Q} \mid \mathbb{P})\right\}
$$

- and if there are $\hat{\theta} \in \mathcal{A}(x)$ and $\hat{\mathbb{Q}} \in \mathcal{M}_{e}{ }^{\prime}$ defined in the following section such that

$$
\frac{1}{\gamma} \ln \mathbb{E}\left[\exp \left(-\gamma\left(V_{T}^{x, \hat{\theta}}-B\right)\right)\right]=-x+\mathbb{E}^{\hat{\mathbb{Q}}}(B)-\frac{1}{\gamma} h(\hat{\mathbb{Q}} \mid \mathbb{P})
$$

then

$$
\begin{aligned}
\frac{\mathrm{d} \hat{\mathbb{Q}}}{\mathrm{d} \mathbb{P}} & =\frac{\exp \left(-\gamma\left(V_{T}^{x, \hat{\theta}}-B\right)\right)}{\mathbb{E} \exp \left(-\gamma\left(V_{T}^{x, \hat{\theta}}-B\right)\right)} \\
V(x, B) & =-x+\sup _{\mathbb{Q} \in \mathcal{M}_{e}^{\prime}}\left\{\mathbb{E}^{\mathbb{Q}}(B)-\frac{1}{\gamma} h(\mathbb{Q} \mid \mathbb{P})\right\} \\
& =\frac{1}{\gamma} \ln \mathbb{E}\left[\exp \left(-\gamma\left(V_{T}^{x, \hat{\theta}}-B\right)\right)\right] .
\end{aligned}
$$

The optimal strategy is

$$
\hat{\theta}_{t}=-\frac{m_{t}}{\gamma \sigma_{t}^{g} S_{t}^{g}}+\frac{z_{t}^{(1)}}{\sigma_{t}^{g} S_{t}^{g}}
$$

with the assumption that the risk premium process $m_{t}$ is bounded, $\left(X^{B}, z\right)$ being the solution of a quadratic BSDE we elaborate in Section 3; the process $\sigma_{t}^{g}$ is defined in Section 1.4.

\subsection{Some reminders about BSDEs}

We recall some facts about backward stochastic differential equations and optimization problems. Let $\mathcal{H}_{T}^{p, d}$ be the space of $\mathbb{R}^{d}$-valued progressively measurable processes $\zeta$ such that:

$$
\mathbb{E}\left[\int_{0}^{T}\left\|\zeta_{t}\right\|^{p} \mathrm{~d} t\right]<\infty
$$

and $\mathcal{H}_{T}^{\infty}\left(\mathbb{R}^{2}\right)$ be the space of progressively measurable $\mathbb{R}^{2}$-valued processes $\zeta$ such that:

$$
\operatorname{ess} \sup _{\Omega \times[0, T]}\left\|\zeta_{t}\right\|<\infty
$$

1. We assume that the mapping $f$ (also called further a generator) from $\Omega \times[0, T] \times \mathbb{R} \times \mathbb{R}^{2}$ into $\mathbb{R}$ is such that $f(\omega, t, 0,0)$ belongs to $\mathcal{H}_{T}^{2,1}$. Moreover, we set the condition: $f$ is uniformly Lipschitz in $(y, z)$, that is there is a constant $\lambda>0$ such that

$$
\left|f\left(t, y^{(1)}, z^{(1)}\right)-f\left(t, y^{(2)}, z^{(2)}\right)\right| \leq \lambda\left(\left|y^{(1)}-y^{(2)}\right|+\left|z^{(1)}-z^{(2)}\right|\right)
$$

for all $\left(y^{(i)}, z^{(i)}\right) \in \mathbb{R} \times \mathbb{R}^{d}, i=1,2$. Also we impose to $f$ to verify $f(t, 0,0) \in \mathcal{H}_{T}^{2,1}$.

2. Then, by a well known result the BSDE

$$
(*)-\mathrm{d} y_{t}=f\left(t, y_{t}, z_{t}\right) \mathrm{d} t-z_{t}^{\prime} \mathrm{d} W_{t}, y_{T}=\phi
$$


with $\phi \in L^{2}(\Omega, \mathcal{F}, \mathbb{P})$ admits a unique solution $(y, z)$ in $\mathcal{H}_{T}^{\infty, 1} \times \mathcal{H}_{T}^{2,2}$.

We recall also the so-called comparison theorem:

3. Suppose that the pair $\left(f^{(i)}, \phi^{(i)}\right), i=1,2$ satisfies the condition in 1 .. We assume also that

$$
\begin{gathered}
f^{(1)}(t, y, z) \geq f^{(2)}(t, y, z), \quad \forall(y, z) \in \mathbb{R} \times \mathbb{R}^{2}, \\
\phi^{(1)} \geq \phi^{(2)} .
\end{gathered}
$$

Let $\left(y^{(i)}, z^{(i)}\right), i=1,2$ denote the solutions of the BSDE $(*)$ with the parameters $(f, \phi)$ to be replaced by the pair $\left(f^{(i)}, \phi^{(i)}\right), i=1,2$ respectively. Then we get

$$
\begin{gathered}
\forall t, y_{t}^{(1)} \geq y_{t}^{(2)} \mathbb{P}-\text { a.s. and } \\
\phi^{(1)} \geq \phi^{(2)}, \phi^{(i)} \in L^{2}(\mathbb{P}), i=1,2 .
\end{gathered}
$$

4. We enounce some results of [14] about quadratic generators and BSDE. That is $f$ to satisfy

$$
\forall(t, y, z) \in[0, T] \times \mathbb{R} \times \mathbb{R}^{2}|f(t, y, z)| \leq \lambda_{1}+\lambda_{2}|y|+\lambda_{3}(|y|)\|z\|^{2}
$$

with $\lambda_{1}, \lambda_{2}$ constants and $\lambda_{3}$ to be a continuous increasing nonnegative function. Let $\phi$ in $L^{\infty}(\mathbb{P})$. Then $(*)$ has a unique solution $(y, z)$ in $\mathcal{H}_{T}^{\infty, 1} \times \mathcal{H}_{T}^{2,2}$ with $y$ continuous mapping. In this case, the comparison principle gives

$$
\forall t \in[0, T] y_{t}^{(1)} \geq y_{t}^{(2)} \mathbb{P}-\text { a.s. }
$$

the assumptions on $\left(f^{(i)}, \phi^{(i)}\right), i=1,2$ being the same as previously, with the supplementary condition: $\phi^{(i)} \in L^{\infty}(\mathbb{P}), i=1,2$.

\subsection{The market model}

Let $(\Omega, \mathcal{F}, \underline{\mathbb{F}}, \mathbb{P})$ be a filtered probability space, equipped with $\underline{\mathbb{F}}=\left(\mathcal{F}_{t}\right)_{0 \leq t \leq T}$ the associated filtration we will precise. Define $\mathcal{F}_{t}^{W}=\sigma\left(W_{s}^{(1)}, W_{s}^{(2)} ; 0 \leq s \leq t\right)$ to be a family of sub- $\sigma$-algebras fields such that $\mathcal{F}_{s}^{W} \subseteq \mathcal{F}_{t}^{W}$, $s \leq t$. Let $\mathcal{N}$ denote the $\mathbb{P}$-null subsets of $\mathcal{F}_{T}^{W}$. Then $\mathcal{F}_{t}=\sigma\left(\mathcal{F}_{t}^{W} \cup \mathcal{N}\right)$ is the augmented filtration of the filtration genrated by the processes $W^{(1)}$ and $W^{(2)}$. The filtration $\mathbb{F}$ is said to satisfy usual conditions. We notice that $\mathcal{F}=\mathcal{F}_{T}$. The processes $W^{(1)}$ and $W^{(2)}$ are two $\mathbb{E}$-adapted independent standard Brownian motions under the probability measure $\mathbb{P}$. $\mathcal{F}_{t}$ represents the information available at time $t ; \mathbb{P}$ is the physical probability under which are modeled $S^{e}$ and $S^{g}$. We assume that the processes $S^{e}$ and $S^{g}$ do satisfy in the time interval $[0, T]$, the stochastic differential equations:

$$
\left\{\begin{array}{l}
\mathrm{d} S_{t}^{e}=S_{t}^{e}\left(\mu_{t}^{e} \mathrm{~d} t+\sigma_{t}^{e}\left(\rho_{t} \mathrm{~d} W_{t}^{(1)}+\sqrt{1-\rho_{t}^{2}} \mathrm{~d} W_{t}^{(2)}\right)\right) \\
\mathrm{d} S_{t}^{g}=S_{t}^{g}\left(\mu_{t}^{g} \mathrm{~d} t+\sigma_{t}^{g} \mathrm{~d} W_{t}^{(1)}\right) .
\end{array}\right.
$$

The model's coefficients $\mu^{e}, \mu^{g}, \sigma^{e}, \sigma^{g}$, and $\rho$ are $\underline{\mathbb{F}}$-adapted and continuous. We add the following assumption on $\sigma^{g}: \forall t \in[0, T] \sigma_{t}^{g} \neq 0 \mathrm{~d} t$-a.s.. To ensure the conditions of integrability, we have:

$$
\int_{0}^{T}\left|\mu_{t}^{i} S_{t}^{i}\right| \mathrm{d} t+\int_{0}^{T}\left|\sigma_{t}^{i} S_{t}^{i}\right|^{2} \mathrm{~d} t<+\infty, \text { for } i=e, g
$$

As the cross variation of the two processes is $\mathrm{d}\left\langle S^{e}, S^{g}\right\rangle_{t}=\rho_{t} \sigma_{t}^{e} \sigma_{t}^{g} S_{t}^{e} S_{t}^{g} \mathrm{~d} t, \rho_{t}$ indicates the level of the correlation between the prices $S^{e}$ and $S^{g}$ during the interval $[t, t+\mathrm{d} t]$. We take the level of correlation to be strictly less than one $\left(\left|\rho_{t}\right|<1,0 \leq t \leq T\right)$. 


\subsection{Martingale probabilities}

Let $\mathbb{Q}$ be a probability measure equivalent to $\mathbb{P}$ on $\mathcal{F}_{T}$. It is well known that there exists two $\underline{\mathbb{F}}$-adapted processes $m$ and $\nu$ such that:

$$
\begin{gathered}
\int_{0}^{T}\left(m_{t}^{2}+\nu_{t}^{2}\right) \mathrm{d} t<+\infty \mathbb{P} \text {-a.s. and } \\
\frac{\mathrm{d} \mathbb{Q}}{\mathrm{d} \mathbb{P}}=\exp \left(\int_{0}^{T} m_{t} \mathrm{~d} W_{t}^{(1)}-\frac{1}{2} \int_{0}^{T} m_{t}^{2} \mathrm{~d} t+\int_{0}^{T} \nu_{t} \mathrm{~d} W_{t}^{(2)}-\frac{1}{2} \int_{0}^{T} \nu_{t}^{2} \mathrm{~d} t\right) .
\end{gathered}
$$

By Girsanov theorem, the processes $\tilde{W}^{(1)}$ and $\tilde{W}^{(2)}$ are defined as follows:

$$
\mathrm{d} \tilde{W}_{t}^{(1)}=\mathrm{d} W_{t}^{(1)}-m_{t} \mathrm{~d} t \text { and } \mathrm{d} \tilde{W}_{t}^{(2)}=\mathrm{d} W_{t}^{(2)}-\nu_{t} \mathrm{~d} t
$$

are two independent standard Brownian motions under the probability $\mathbb{Q}$.

As we have in our general model a Brownian filtration, we will use in the sequel only a previsible representation property for a $\mathbb{Q}$-local martingale adapted to the filtration $\left(\mathcal{F}_{t}\right)$, where $\mathbb{Q}$ is a probability equivalent to $\mathbb{P}$. The change of Brownian motion induced by the change of probability doesn't allow the use of the representation property for martingales adapted to the filtration $\left(\mathcal{F}_{t}\right)$. Nevertheless, we state the following decomposition theorem of such local martingales:

Theorem 1.1. Let $\overline{\mathbb{Q}}$ be a probability equivalent to $\mathbb{P}$. Let $\bar{W}=\left(\bar{W}^{(1)}, \bar{W}^{(2)}\right)$ be a $\overline{\mathbb{Q}}$-standard brownian motion issued from $W=\left(W^{(1)}, W^{(2)}\right)$ by Girsanov theorem. Let $N$ be a $\overline{\mathbb{Q}}$-local martingale process. Then $N$ admits a version such that there exists a $\mathbb{R}^{2}$-valued process $h=\left(\tilde{h}^{(1)}, \tilde{h}^{(2)}\right) \underline{\mathbb{F}}$-adapted, $\int_{0}^{T}\left(\left(\tilde{h}_{t}^{(1)}\right)^{2}+\left(\tilde{h}_{t}^{(2)}\right)^{2}\right) \mathrm{d} t<+\infty$ $\mathbb{P}$-a.s.:

$$
N_{t}=N_{0}+\int_{0}^{t} \tilde{h}_{s}^{(1)} \mathrm{d} \bar{W}_{s}^{(1)}+\int_{0}^{t} \tilde{h}_{s}^{(2)} \mathrm{d} \bar{W}_{s}^{(2)}, \quad 0 \leq t \leq T .
$$

Moreover, if $N$ is a square integrable $\overline{\mathbb{Q}}$-matingale, then

$$
\mathbb{E}^{\overline{\mathbb{Q}}}\langle N, N\rangle_{T}<+\infty
$$

The proof is in appendix.

A necesary and sufficient condition for the process $S^{g}$ to be a local martingale under $\mathbb{Q}$ is:

$$
\mu_{t}^{g}+\sigma_{t}^{g} m_{t}=0
$$

and we get

$$
\frac{\mathrm{d} S_{t}^{g}}{S_{t}^{g}}=\sigma_{t}^{g} \mathrm{~d} \tilde{W}_{t}^{(1)}
$$

From now we suppose the equality (1.3) to be satisfied, i.e. $m_{t}:=-\frac{\mu_{t}^{g}}{\sigma_{t}^{g}}, \quad 0 \leq t \leq T$.

The condition $S^{g}$ local martingale under $\mathbb{Q}$ fixes the value of $m$ in (1.1). Consequently, the martingale measures on $\mathcal{F}_{T}$ are parametrized by a process $\nu$. More precisely:

Let $\left(\xi_{t}^{\nu}\right)_{0 \leq t \leq T}$ be the real process defined by the stochastic differential equation: $\mathrm{d} \xi_{t}^{\nu}=\xi_{t}^{\nu} m_{t} \mathrm{~d} W_{t}^{(1)}+$ $\left.\nu_{t} \mathrm{~d} W_{t}^{(2)}\right), \xi_{0}^{\nu}=1$. We have $\mathbb{P}$-a.s.

$$
\xi_{t}^{\nu}=\exp \left(\int_{0}^{t} m_{s} \mathrm{~d} W_{s}^{(1)}-\frac{1}{2} \int_{0}^{t} m_{s}^{2} \mathrm{~d} s+\int_{0}^{t} \nu_{s} \mathrm{~d} W_{s}^{(2)}-\frac{1}{2} \int_{0}^{t} \nu_{s}^{2} \mathrm{~d} s\right), 0 \leq t \leq T .
$$


Define then the set $\mathcal{K}$ of $\underline{\mathbb{F}}$-adapted and real processes $\nu$ such that $\int_{0}^{T} \nu_{t}^{2} \mathrm{~d} t<+\infty \mathbb{P}$-a.s. and $\left(\xi_{t}^{\nu}\right)_{0 \leq t \leq T}$ is a $\mathbb{P}$-martingale. Therefore, given any $\nu \in \mathcal{K}$, we can define a probability measure $\mathbb{P}^{\nu}$ equivalent to $\mathbb{P}$ by $\mathrm{d} \mathbb{P}^{\nu}=\xi_{T}^{\nu} d \mathbb{P}$. We have the following characterization of the set $\mathcal{M}_{e}$ :

$$
\mathcal{M}_{e}=\left\{\mathbb{P}^{\nu} \mid \nu \in \mathcal{K}\right\}
$$

The new probability measure

$$
\mathbb{P}^{\nu}(A)=\int_{A} \xi_{T}^{\nu} \mathrm{d} \mathbb{P}, \quad A \text { belongs to } \mathcal{F}_{T}
$$

is such that $\mathbb{P}^{\nu}$ is absolutely continuous on $\mathcal{F}_{T}$ with respect to $\mathbb{P}\left(\mathbb{P}^{\nu} \ll \mathbb{P}\right)$. Moreover, since $\xi_{T}^{\nu}>0 \mathbb{P}$-a.s., then the two measures are equivalent $\left(\mathbb{P}^{\nu} \sim \mathbb{P}\right)$.

Now we construct our financial portfolio. We have an investor who starts with some initial endowment $x$ and invests it in the bond and in the proxy asset. Let $\left(V_{t}\right)_{0 \leq t \leq T}$ be the investor's wealth process. It will be the hedging portfolio when the investor holds an option. Then, in our model the portfolio is constructed upon the assets of prices $S^{g}$ and $S^{0} \equiv 1$. Thus, its value at time $t$ is

$$
V_{t}=\eta_{t} S_{t}^{0}+\theta_{t} S_{t}^{g}=\eta_{t}+\theta_{t} S_{t}^{g}
$$

$\eta_{t}$ and $\theta_{t}$ being respectively the quantities of assets of prices $S^{0}$ and $S^{g}$ detained at time $t$ by the investor in the portfolio. We make the assumption that the portfolio is self-financing; that is

$$
V_{t}=V_{t}^{x, \theta}=x+\int_{0}^{t} \theta_{s} \mathrm{~d} S_{s}^{g}
$$

with $\theta$ an element of

$$
L\left(S^{g}\right)=\left\{\theta \underline{\mathbb{E}} \text {-adapted } \mid \int_{0}^{T} \theta_{t}^{2} \mathrm{~d}\left\langle S^{g}, S^{g}\right\rangle_{t}<+\infty \mathbb{P}-\text { a.s. }\right\} .
$$

We will precise latter the space of admissible strategies. Define a contingent claim to be an $\mathcal{F}_{T}$-measurable random variable.

To evaluate and hedge the contingent claim $H$ by an exponential utility function, it is necessary to compute the quantities

$$
v(x+p, H)=\sup _{\theta} \mathbb{E}\left[-\mathrm{e}^{-\gamma\left(V_{T}^{x+p, \theta}-H\right)}\right] \text { and } v(x, 0)=\sup _{\theta} \mathbb{E}\left[-\mathrm{e}^{-\gamma V_{T}^{x, \theta}}\right],
$$

where $p$ is the option's price as $x$ is the initial endowment when the investors plans not to sell the option.

Hence we must choose at the beginning the space of admissible strategies $\Theta(x)=\mathcal{A}(x)$, upon which a supremum is attained.

\subsection{The set of admissible strategies}

The set of admissible strategies must allow:

- to avoid arbitrage opportunities on the financial market $\left\{S^{0}, S^{g}\right\}$;

- to ensure the existence of $\mathbb{E} \mathrm{e}^{-\gamma V_{T}^{x, \theta}}$ and $\mathbb{E}\left[\mathrm{e}^{-\gamma\left(V_{T}^{x, \theta}-H\right)}\right]$;

- to guarantee the existence of admissible optimal solutions to the problems

$$
\left(\mathcal{P}_{H}\right) \sup _{\theta} \mathbb{E}\left[-\mathrm{e}^{-\gamma\left(V_{T}^{x, \theta}-H\right)}\right] \text { and }\left(\mathcal{P}_{0}\right) \sup _{\theta} \mathbb{E}\left[-\mathrm{e}^{-\gamma V_{T}^{x, \theta}}\right]
$$


The concept of hedging strategies is introduced in order to allow the solution of the contingent claim valuation problem. Letting

$$
\mathcal{A}_{b}(x):=\left\{\theta \in L\left(S^{g}\right): \exists a_{\theta, x} \in \mathbb{R}, V_{t}^{x, \theta} \geq a_{\theta, x} \text { P-a.s. } \forall t, 0 \leq t \leq T\right\},
$$

we assume that

$$
\mathcal{M}_{e} \neq \emptyset \text {. }
$$

Henceforth, for $\theta \in \mathcal{A}_{b}(x), V^{x, \theta}$ is lower bounded. Moreover $V^{x, \theta}$ is a $\mathbb{Q}$-local martingale for all $\mathbb{Q}$ element of $\mathcal{M}_{e}$. Finally $V^{x, \theta}$ is a $\mathbb{Q}$-supermartingale according to Fatou's lemma.

Proposition 1.1. There are no arbitrage opportunities on the financial market $\left\{S^{0}, S^{g}\right\}$ when the strategies are in $\mathcal{A}_{b}(x)$.

Proof. Indeed, assume that there are arbitrage opportunities for a hedging strategy $\theta \in \mathcal{A}_{b}(x)$ : that is there exist $x \in \mathbb{R}$ and $\theta \in \mathcal{A}_{b}(x)$ :

$$
x \leq 0 \text { and } V_{T}^{x, \theta} \geq 0 \mathbb{P} \text {-a.s., } \mathbb{P}\left(V_{T}^{x, \theta}>0\right)>0 .
$$

$\theta \in \mathcal{A}_{b}(x)$ implies $V^{x, \theta} \mathbb{Q}$-supermartingale; $\mathbb{E}^{\mathbb{Q}}\left[V_{T}^{x, \theta}\right] \leq x \leq 0 ;$ $\frac{\mathrm{d} \mathbb{Q}}{\mathrm{d} \mathbb{P}} V_{T}^{x, \theta} \geq 0 \mathbb{P}$-a.s. implies $\mathbb{E}^{\mathbb{Q}}\left[V_{T}^{x, \theta}\right] \geq 0$. Hence $\mathbb{E}^{\mathbb{Q}}\left[V_{T}^{x, \theta}\right]=0 ;$ $V_{T}^{x, \theta} \geq 0 \mathbb{P}$-a.s., $\mathbb{P}\left(V_{T}^{x, \theta}>0\right)>0$ implies $\mathbb{E}^{\mathbb{Q}}\left[V_{T}^{x, \theta}\right]=\mathbb{E}^{\mathbb{Q}}\left[V_{T}^{x, \theta} 1_{\left\{V_{T}^{x, \theta}>0\right\}}\right]>0$.

This is impossible. Hence there are no arbitrage opportunities on the market $\left\{S^{0}, S^{g}\right\}$ for $\theta \in \mathcal{A}_{b}(x)$.

We choose a space of admissible strategies which contains the strategies $\theta$ such that the wealth process $V^{x, \theta}$ is not necessarily lower bounded and which could guarantee the existence of an optimal solution in that space.

We then define the set:

$$
\mathcal{A}(x):=\left\{\theta \in L\left(S^{g}\right) \mid \exists\left(\theta_{n}\right)_{n} \in \mathcal{A}_{b}(x) \text { such that } \mathrm{e}^{-\gamma\left(V_{T}^{x, \theta_{n}}-H\right)} \stackrel{L^{1}(\mathbb{P})}{\rightarrow} \mathrm{e}^{-\gamma\left(V_{T}^{x, \theta}-H\right)}\right\} .
$$

We get in particular $\forall \theta \in \mathcal{A}(x)$, exp $-\gamma\left(V_{T}^{x, \theta}-H\right) \in L^{1}(\mathbb{P})$. We need $\mathrm{e}^{\gamma H} \in L^{1}(\mathbb{P})$; this hypothesis holds for instance when $H$ is a bounded random variable.

The set of admissible strategies for a given initial wealth $x$ is $\mathcal{A}(x)$. It contains $\mathcal{A}_{b}(x)$ as well as some strategies $\theta$ such that $V^{x, \theta}$ is non necessarily lower bounded.

\section{Formulation of the DUAL PROBlem}

Let $h(\mathbb{Q} \mid \mathbb{P})$ be the relative entropy of a probability measure $\mathbb{Q}$ with respect to $\mathbb{P}$;

$$
h(\mathbb{Q} \mid \mathbb{P})=\left\{\begin{array}{cc}
\mathbb{E}\left[\frac{\mathrm{d} \mathbb{Q}}{\mathrm{d} \mathbb{P}} \ln \left(\frac{\mathrm{d} \mathbb{Q}}{\mathrm{d} \mathbb{P}}\right)\right] & \text { if } \mathbb{Q} \ll \mathbb{P} \\
+\infty & \text { otherwise. }
\end{array}\right.
$$

Let $B$ be a random variable.

We denote by $\mathbb{E}^{\mathbb{Q}}(\cdot)$ the expectation operator with respect to the probability measure $\mathbb{Q}$.

Let us define

$$
\mathcal{M}^{B}:=\left\{\mathbb{Q} \ll \mathbb{P} \mid h(\mathbb{Q} \mid \mathbb{P})<+\infty \text { and } \mathbb{E}^{\mathbb{Q}}(B)<+\infty\right\} .
$$


We will show the following lemma which will be useful to formulate the dual problem of $\sup _{\theta} \mathbb{E}\left[-\mathrm{e}^{-\gamma\left(V_{T}^{x, \theta}-H\right)}\right]$ :

Lemma 2.1. For B random variable almost surely finite,

$$
\ln \left(\mathbb{E} \mathrm{e}^{B}\right)=\sup _{\mathbb{Q} \in \mathcal{M}^{B}}\left\{\mathbb{E}^{\mathbb{Q}}(B)-h(\mathbb{Q} \mid \mathbb{P})\right\} .
$$

Moreover if $\mathbb{Q} \in \mathcal{M}^{B}$, we get $\ln \left(\mathbb{E}^{B}\right)=\mathbb{E}^{\mathbb{Q}}(B)-h(\mathbb{Q} \mid \mathbb{P})$ if and only if

$$
\mathbb{E}\left(B \mathrm{e}^{B}\right)<\infty \text { and } \frac{\mathrm{d} \mathbb{Q}}{\mathrm{d} \mathbb{P}}=\frac{e^{B}}{\mathbb{E} e^{B}} .
$$

Proof. If we take $\mathbb{Q} \in \mathcal{M}^{B}$, then $\mathbb{E}^{\mathbb{Q}}(B)-h(\mathbb{Q} \mid \mathbb{P})=\mathbb{E}^{\mathbb{Q}}\left[\ln \left(\frac{e^{B}}{d \mathbb{Q} / d \mathbb{P}}\right)\right]$. Hence, by Jensen's inequality, we have, for $\mathbb{Q} \in \mathcal{M}^{B}$,

$$
\mathbb{E}^{\mathbb{Q}}(B)-h(\mathbb{Q} \mid \mathbb{P})=\mathbb{E}^{\mathbb{Q}}\left[\ln \left(\frac{e^{B}}{\mathrm{~d} \mathbb{Q} / \mathrm{d} \mathbb{P}}\right)\right]=\ln \mathbb{E}\left(e^{B} I_{\left\{\frac{\mathrm{d}}{\mathrm{dP}}>0\right\}}\right) \leq \ln \left(\mathbb{E}^{B}\right),
$$

where $I_{A}(\omega)$ is the characteristic function of the set $A$; that is $I_{A}(\omega)=1$ if $\omega$ belongs to $A$, or 0 otherwise.

But, since $x \longmapsto \ln x$ is stictly concave, there is equality only if $\frac{e^{B}}{\mathrm{~d} \mathbb{Q} / \mathrm{dP}}=$ constant $\mathbb{Q}$-a.s.

Hence $\frac{\mathrm{d} \mathbb{Q}}{\mathrm{dP}}=\frac{\mathrm{e}^{B}}{\mathbb{E}^{B}}$ a.s., and there is uniqueness if the supremum is attained. It is the case if $B$ is almost surely finite.

To show the equality: $\ln \left(\mathbb{E} e^{B}\right)=\sup _{\mathbb{Q} \in \mathcal{M}^{B}}\left\{\mathbb{E}^{\mathbb{Q}}(B)-h(\mathbb{Q} \mid \mathbb{P})\right\}$, let us introduce the sequence of probability measures $\mathbb{Q}_{n}$ defined by

$$
\frac{\mathrm{d} \mathbb{Q}_{n}}{\mathrm{~d} \mathbb{P}}=\frac{\mathrm{e}^{B} I_{\{|B| \leq n\}}}{\mathbb{E}\left(\mathrm{e}^{B} I_{\{|B| \leq n\}}\right)} .
$$

Since $|B|<\infty$ a.s., $\mathbb{Q}_{n}$ is well defined for a sufficiently large $n$ and $\mathbb{Q}_{n} \in \mathcal{M}^{B}$.

We obtain $\mathbb{E}^{\mathbb{Q}_{n}}(B)-h\left(\mathbb{Q}_{n} \mid \mathbb{P}\right)=\ln \mathbb{E}\left(\mathrm{e}^{B} I_{\{|B| \leq n\}}\right)$.

By monotone convergence theorem, we have the equality; whence the desired result.

We recall that $0 \leq t \leq T$,

$$
\xi_{t}^{\nu}=\exp \left(\int_{0}^{t} m_{s} \mathrm{~d} W_{s}^{(1)}-\frac{1}{2} \int_{0}^{t} m_{s}^{2} \mathrm{~d} s+\int_{0}^{t} \nu_{s} \mathrm{~d} W_{s}^{(2)}-\frac{1}{2} \int_{0}^{t} \nu_{s}^{2} \mathrm{~d} s\right) .
$$

We recall also the characterization of the set of equivalent martingale measures $\mathcal{M}_{e}$. Indeed, we have in view to formulate the dual problem whose the optimal solution is in a subset of $\mathcal{M}_{e}$ under certain conditions we will precise in the sequel. Then

$$
\begin{gathered}
\mathcal{M}_{e}=\left\{\mathbb{P}^{\nu} \mid \frac{\mathrm{d} \mathbb{P}^{\nu}}{\mathrm{d} \mathbb{P}}=\xi_{T}^{\nu}, \nu \mathcal{F}-\right.\text { adapted such that } \\
\left.\int_{0}^{T}\left(m_{t}^{2}+\nu_{t}^{2}\right) \mathrm{d} t+\infty \text { a.s. and } \xi^{\nu} \mathbb{P} \text { - martingale }\right\} .
\end{gathered}
$$

We get

$$
\ln \left(\frac{\mathrm{d} \mathbb{P}^{\nu}}{\mathrm{d} \mathbb{P}}\right)=\int_{0}^{T} m_{t} \mathrm{~d} W_{t}^{(1)}-\frac{1}{2} \int_{0}^{T} m_{t}^{2} \mathrm{~d} t+\int_{0}^{T} \nu_{t} \mathrm{~d} W_{t}^{(2)}-\frac{1}{2} \int_{0}^{T} \nu_{t}^{2} \mathrm{~d} t .
$$


Under the martingale measure $\mathbb{P}^{\nu}$,

$$
\ln \left(\frac{\mathrm{d} \mathbb{P}^{\nu}}{\mathrm{d} \mathbb{P}}\right)=\int_{0}^{T} m_{t} \mathrm{~d} \tilde{W}_{t}^{(1)}+\frac{1}{2} \int_{0}^{T} m_{t}^{2} \mathrm{~d} t+\int_{0}^{T} \nu_{t} \mathrm{~d} \tilde{W}_{t}^{(2)}+\frac{1}{2} \int_{0}^{T} \nu_{t}^{2} \mathrm{~d} t
$$

We define the process $M_{t}:=\xi_{t}^{\nu}=\mathbb{E}\left(\frac{\mathrm{d} \mathbb{P}^{\nu}}{\mathrm{dP}} \mid \mathcal{F}_{t}\right)$ which will be frequently used in the proofs throughout this section and in the following.

We define now the set

$$
\mathcal{M}_{e}^{\prime}:=\left\{\mathbb{Q}=\mathbb{P}^{\nu} \mid \mathbb{E}^{\mathbb{P}^{\nu}} \int_{0}^{T}\left(m_{t}^{2}+\nu_{t}^{2}\right) \mathrm{d} t<+\infty\right\}
$$

and we assume that

$$
\mathcal{M}_{e}{ }^{\prime} \neq \emptyset .
$$

The subset $\mathcal{M}_{e}{ }^{\prime}$ of $\mathcal{M}_{e}$ is the space upon which we want to find the solution of the dual problem that remains to define. First we characterize $\mathcal{M}_{e}{ }^{\prime}$ by showing notably that it is the set of probabilities with finite entropy. We have the following theorem:

Theorem 2.1. A characterization of the set $\mathcal{M}_{e}{ }^{\prime}$ is

$$
\mathcal{M}_{e}^{\prime}=\left\{\mathbb{Q}=\mathbb{P}^{\nu} \mid h\left(\mathbb{P}^{\nu} \mid \mathbb{P}\right)<+\infty\right\}
$$

Moreover we have, for all $\mathbb{P}^{\nu} \in \mathcal{M}_{e}^{\prime}$,

$$
h\left(\mathbb{P}^{\nu} \mid \mathbb{P}\right)=\frac{1}{2} \mathbb{E}^{\mathbb{P}^{\nu}} \int_{0}^{T}\left(m_{t}^{2}+\nu_{t}^{2}\right) \mathrm{d} t
$$

Proof. If $\mathbb{P}^{\nu} \in \mathcal{M}_{e}{ }^{\prime}, \mathbb{E}^{\mathbb{P}^{\nu}} \int_{0}^{T}\left(m_{t}^{2}+\nu_{t}^{2}\right) \mathrm{d} t<+\infty$. Hence the processes $\int_{0} m_{s} \mathrm{~d} \tilde{W}_{s}^{(1)}$ and $\int_{0} \nu_{s} \mathrm{~d} \tilde{W}_{s}^{(2)}$ are martingales under the martingale measure $\mathbb{P}^{\nu}$.

But, according to $(2.2)$, we have

$$
h\left(\mathbb{P}^{\nu} \mid \mathbb{P}\right)=\mathbb{E}^{\mathbb{P}^{\nu}} \ln \frac{\mathrm{d} \mathbb{P}^{\nu}}{\mathrm{d} \mathbb{P}}=\mathbb{E}^{\mathbb{P}^{\nu}}\left(\int_{0}^{T} m_{t} \mathrm{~d} \tilde{W}_{t}^{(1)}+\frac{1}{2} \int_{0}^{T} m_{t}^{2} \mathrm{~d} t+\int_{0}^{T} \nu_{t} \mathrm{~d} \tilde{W}_{t}^{(2)}+\frac{1}{2} \int_{0}^{T} \nu_{t}^{2} \mathrm{~d} t\right) .
$$

Henceforth $h\left(\mathbb{P}^{\nu} \mid \mathbb{P}\right)=\frac{1}{2} \mathbb{E}^{\mathbb{P}^{\nu}} \int_{0}^{T}\left(m_{t}^{2}+\nu_{t}^{2}\right) \mathrm{d} t<+\infty$.

Conversely, there is an equivalence between the assertions:

1. $h\left(\mathbb{P}^{\nu} \mid \mathbb{P}\right)<+\infty$,

2. $\mathbb{E}\left(M_{T} \ln M_{T}\right)<+\infty$.

Indeed, the function $\varphi: x \mapsto x \ln x$ is convex on $[0,+\infty)$ and admits a unique minimum in $x=\frac{1}{e}: \forall x \geq$ $0, x \ln x \geq-\frac{1}{e}$.

We set $\tau_{n}=\inf \left\{t \in[0, T] \mid \int_{0}^{t}\left(m_{s}^{2}+\nu_{s}^{2}\right) \mathrm{d} s \geq n\right\}, n \geq 1 .\left(\tau_{n}\right)_{n \geq 1}$ is a sequence of stopping times converging towards infinity. We adopt the convention $\inf \emptyset=+\infty$.

We obtain

$$
\begin{aligned}
\mathbb{E} M_{T} \ln M_{T \wedge \tau_{n}} & =\mathbb{E}\left(\mathbb{E}\left(M_{T} \ln M_{T \wedge \tau_{n}} \mid \mathcal{F}_{T \wedge \tau_{n}}\right)\right) \\
& =\mathbb{E} M_{T \wedge \tau_{n}} \ln M_{T \wedge \tau_{n}} .
\end{aligned}
$$


Hence

$$
\begin{aligned}
\mathbb{E} M_{T \wedge \tau_{n}} \ln M_{T \wedge \tau_{n}} & =\mathbb{E} M_{T \wedge \tau_{n}}\left(\int_{0}^{T \wedge \tau_{n}} m_{t} \mathrm{~d} W_{t}^{(1)}+\int_{0}^{T \wedge \tau_{n}} \nu_{t} \mathrm{~d} W_{t}^{(2)}-\frac{1}{2} \int_{0}^{T \wedge \tau_{n}}\left(m_{t}^{2}+\nu_{t}^{2}\right) \mathrm{d} t\right) \\
& =\mathbb{E}^{\mathbb{P}^{\nu}}\left(\int_{0}^{T \wedge \tau_{n}} m_{t} \mathrm{~d} \tilde{W}_{t}^{(1)}+\int_{0}^{T \wedge \tau_{n}} \nu_{t} \mathrm{~d} \tilde{W}_{t}^{(2)}+\frac{1}{2} \int_{0}^{T \wedge \tau_{n}}\left(m_{t}^{2}+\nu_{t}^{2}\right) \mathrm{d} t\right) \\
& =\frac{1}{2} \mathbb{E}^{\mathbb{P}^{\nu}} \int_{0}^{T \wedge \tau_{n}}\left(m_{t}^{2}+\nu_{t}^{2}\right) \mathrm{d} t
\end{aligned}
$$

The sequence $\tau_{n}$ being increasing, we get by monotone convergence theorem

$$
\begin{aligned}
\lim _{n \longrightarrow \infty} \mathbb{E} M_{T \wedge \tau_{n}} \ln M_{T \wedge \tau_{n}} & =\sup _{n \geq 1} \mathbb{E} M_{T \wedge \tau_{n}} \ln M_{T \wedge \tau_{n}} \\
& =\frac{1}{2} \mathbb{E}^{\mathbb{P}^{\nu}} \int_{0}^{T}\left(m_{t}^{2}+\nu_{t}^{2}\right) \mathrm{d} t .
\end{aligned}
$$

But conditional Jensen's inequality yields

$$
M_{T \wedge \tau_{n}} \ln M_{T \wedge \tau_{n}} \leq \mathbb{E}\left(M_{T} \ln M_{T} \mid \mathcal{F}_{T \wedge \tau_{n}}\right)
$$

By hypothesis, $M_{T} \ln M_{T}$ is integrable.

Hence the sequence $\left(M_{T \wedge \tau_{n}} \ln M_{T \wedge \tau_{n}}\right)_{n}$ is uniformly integrable, and we get

$$
\begin{aligned}
\lim _{n \rightarrow \infty} \mathbb{E} M_{T \wedge \tau_{n}} \ln M_{T \wedge \tau_{n}} & =\mathbb{E}\left(M_{T} \ln M_{T}\right) \\
& =\frac{1}{2} \mathbb{E}^{\mathbb{P}^{\nu}} \int_{0}^{T \wedge \tau_{n}}\left(m_{t}^{2}+\nu_{t}^{2}\right) \mathrm{d} t<+\infty
\end{aligned}
$$

For $\mathbb{Q}=\mathbb{P}^{\nu} \in \mathcal{M}_{e}{ }^{\prime}$, the entropy with respect to $\mathbb{P}$ is

$$
h(\mathbb{Q} \mid \mathbb{P})=\mathbb{E}^{\mathbb{Q}} \ln \left(\frac{\mathrm{d} \mathbb{Q}}{\mathrm{d} \mathbb{P}}\right)=\mathbb{E}^{\mathbb{Q}}\left(\frac{1}{2} \int_{0}^{T} m_{t}^{2} \mathrm{~d} t+\frac{1}{2} \int_{0}^{T} \nu_{t}^{2} \mathrm{~d} t\right) .
$$

We will next formulate the dual problem for an investor having an initial wealth $x$ as mentionned in Section 1 . The agent invests in the construction of a hedging portfolio $V^{x, \theta}$ and in the same time sells a contingent claim $H$ at the date $t=0$. We suppose that the price of the option of payoff $H$ is included in the initial wealth. Hence the agent's program is:

We set

$$
\begin{gathered}
\left(\mathcal{P}_{H}\right) \quad v(x+p, H):=\sup _{\theta \in \mathcal{A}(x+p)} \mathbb{E}\left[-\mathrm{e}^{\left.-\gamma\left(V_{T}^{x+p, \theta}-H\right)\right]}\right. \\
=-\inf _{\theta \in \mathcal{A}(x+p)} \mathbb{E}\left[\mathrm{e}^{\left.-\gamma\left(V_{T}^{x+p, \theta}-H\right)\right]}\right.
\end{gathered}
$$

$$
\left(\mathcal{P}_{0}\right) \quad v(x, 0):=\sup _{\theta \in \mathcal{A}(x)} \mathbb{E}\left[-\mathrm{e}^{-\gamma V_{T}^{x, \theta}}\right]=-\inf _{\theta \in \mathcal{A}(x)} \mathbb{E}\left[\mathrm{e}^{-\gamma V_{T}^{x, \theta}}\right]
$$

$\left(\mathcal{P}_{0}\right)$ is the program of the agent having the initial wealth $x$, and invests only in the construction of a portfolio $\theta$. In the two cases the agent is maximizing the expected utility $v$. Then, the indifference price $(c f$. [8]) is defined by the equality:

$$
v(x+p, H)=v(x, 0)
$$


or equivalently,

From now, we set

$$
\frac{1}{\gamma} \ln [-v(x+p, H)]=\frac{1}{\gamma} \ln [-v(x, 0)] .
$$

$$
V(x, H):=\frac{1}{\gamma} \ln [-v(x, H)]
$$

Henceforth, we will try to compute $V(x, H)$ in order to get a fair characterization of the optimal hedging $\hat{\theta}$ and the price $p$.

Let us calculate now

$$
V(x, H)=\inf _{\theta \in \mathcal{A}(x)} \frac{1}{\gamma} \ln \mathbb{E}\left[\exp \left(-\gamma\left(V_{T}^{x, \theta}-H\right)\right)\right] .
$$

Let us exhibit another set of martingale measures which will fully characterize the dual problem.

Let $\theta$ be in $\mathcal{A}_{b}(x)$. We define the set

$$
\mathcal{M}_{\theta}^{\prime}:=\left\{\mathbb{Q} \ll \mathbb{P} \mid V^{x, \theta} \mathbb{Q} \text {-integrable and } h(\mathbb{Q} \mid \mathbb{P})<+\infty\right\} .
$$

By using Lemma 2.1, if $\theta \in \mathcal{A}_{b}(x)$

$$
\frac{1}{\gamma} \ln \mathbb{E}\left[\exp \left(-\gamma\left(V_{T}^{x, \theta}-H\right)\right)\right]=\sup _{\mathbb{Q} \in \mathcal{M}_{\theta}^{\prime}}\left\{\mathbb{E}^{\mathbb{Q}}\left[-V_{T}^{x, \theta}+H\right]-\frac{1}{\gamma} h(\mathbb{Q} \mid \mathbb{P})\right\}
$$

Indeed, if $\mathbb{Q}$ is not in the set $\mathcal{M}_{\theta}^{\prime}, h(\mathbb{Q} \mid \mathbb{P})=+\infty$. As $H$ is almost surely finite, and as $\theta \in \mathcal{A}_{b}(x)$ implies

$$
\mathbb{E}^{\mathbb{Q}}\left[V_{T}^{x, \theta}\right] \leq x
$$

hence we have

$$
\begin{aligned}
\frac{1}{\gamma} \sup _{\mathbb{Q} \in \mathcal{M}_{\theta}^{\prime}}\left\{\mathbb{E}^{\mathbb{Q}}\left[-\gamma\left(-V_{T}^{x, \theta}+H\right)\right]-h(\mathbb{Q} \mid \mathbb{P})\right\} & \geq \frac{1}{\gamma} \sup _{\mathbb{Q} \in \mathcal{M}_{e}^{\prime}}\left\{\mathbb{E}^{\mathbb{Q}}\left[-\gamma\left(-V_{T}^{x, \theta}+H\right)\right]-h(\mathbb{Q} \mid \mathbb{P})\right\} \\
& \geq \sup _{\mathbb{Q} \in \mathcal{M}_{e}^{\prime}}\left\{\mathbb{E}^{\mathbb{Q}}\left[-\left(-V_{T}^{x, \theta}+H\right)\right]-\frac{1}{\gamma} h(\mathbb{Q} \mid \mathbb{P})\right\} \\
& \geq-x+\sup _{\mathbb{Q} \in \mathcal{M}_{e}^{\prime}}\left\{\mathbb{E}^{\mathbb{Q}}(H)-\frac{1}{\gamma} h(\mathbb{Q} \mid \mathbb{P})\right\} .
\end{aligned}
$$

Consequently,

$$
\begin{aligned}
V(x, H) & =\inf _{\theta \in \mathcal{A}(x)} \frac{1}{\gamma} \ln \mathbb{E}\left[\exp \left(-\gamma\left(V_{T}^{x, \theta}-H\right)\right)\right] \\
& =\inf _{\theta \in \mathcal{A}_{b}(x)} \frac{1}{\gamma} \ln \mathbb{E}\left[\exp \left(-\gamma\left(V_{T}^{x, \theta}-H\right)\right)\right]
\end{aligned}
$$

Hence $V(x, H) \geq-x+\sup _{\mathbb{Q} \in \mathcal{M}_{e}^{\prime}}\left\{\mathbb{E}^{\mathbb{Q}}(H)-\frac{1}{\gamma} h(\mathbb{Q} \mid \mathbb{P})\right\}$.

If we can find $\hat{\theta} \in \mathcal{A}(x)$ and $\hat{\mathbb{Q}} \in \mathcal{M}_{e}^{\prime}$ such that

$$
\frac{1}{\gamma} \ln \mathbb{E}\left[\exp \left(-\gamma\left(V_{T}^{x, \hat{\theta}}-H\right)\right)\right]=-x+\mathbb{E}^{\hat{\mathbb{Q}}}(H)-\frac{1}{\gamma} h(\hat{\mathbb{Q}} \mid \mathbb{P}),
$$

the previous inequality is an equality. 
We first study the problem:

$$
\sup _{\mathbb{Q} \in \mathcal{M}_{e^{\prime}}}\left\{\mathbb{E}^{\mathbb{Q}}(H)-\frac{1}{\gamma} h(\mathbb{Q} \mid \mathbb{P})\right\}=\sup _{\mathbb{P}^{\nu} \in \mathcal{M}_{e^{\prime}}}\left\{\mathbb{E}^{\mathbb{P}^{\nu}}(H)-\frac{1}{2 \gamma} \mathbb{E}^{\mathbb{P}^{\nu}} \int_{0}^{T}\left(m_{t}^{2}+\nu_{t}^{2}\right) \mathrm{d} t\right\} .
$$

We will solve the formulated control problem in the following section.

\section{Properties of the optimal dual process}

Denote by $\mathcal{K}_{e}{ }^{\prime}$ the set of progressively measurable processes $\nu$ such that $\mathbb{P}^{\nu} \in \mathcal{M}_{e}{ }^{\prime}$. We define the process $\left(M_{t}^{\nu}\right)_{0 \leq t \leq T}$ by

$$
M_{t}^{\nu}:=\mathbb{E}^{\mathbb{P}^{\nu}}\left[H-\frac{1}{2 \gamma} \int_{0}^{T}\left(m_{t}^{2}+\nu_{t}^{2}\right) \mathrm{d} t \mid \mathcal{F}_{t}\right] .
$$

$M^{\nu}$ is a martingale process under $\mathbb{P}^{\nu}$.

According to the Theorem 1.1 there is a process $z^{\nu} \quad \mathcal{F}$-adapted with values in $\mathbb{R}^{2}$ such that $\int_{0}^{T}\left(\left(z_{t}^{1, \nu}\right)^{2}+\right.$ $\left.\left(z_{t}^{2, \nu}\right)^{2}\right) \mathrm{d} t<+\infty \mathbb{P}$-a.s. and:

$$
M_{t}^{\nu}:=M_{0}^{\nu}+\int_{0}^{t}\left(z_{s}^{\nu}\right)^{\prime} \mathrm{d} \tilde{W}_{s}=M_{0}^{\nu}+\int_{0}^{t} z_{s}^{1, \nu} \mathrm{d} \tilde{W}_{s}^{(1)}+\int_{0}^{t} z_{s}^{2, \nu} \mathrm{d} \tilde{W}_{s}^{(2)}, \quad 0 \leq t \leq T
$$

Let $\left(X_{t}^{H, \nu}\right)_{0 \leq t \leq T}$ be the process defined by:

$$
X_{t}^{H, \nu}:=\mathbb{E}^{\mathbb{P}^{\nu}}\left[H-\frac{1}{2 \gamma} \int_{t}^{T}\left(m_{s}^{2}+\nu_{s}^{2}\right) \mathrm{d} s \mid \mathcal{F}_{t}\right]
$$

and

It is clear that

$$
X_{t}^{H}:=\operatorname{ess} \sup _{\nu} X_{t}^{H, \nu}
$$

$$
X_{t}^{H, \nu}=\frac{1}{2 \gamma} \int_{0}^{t}\left(m_{s}^{2}+\nu_{s}^{2}\right) \mathrm{d} s+M_{t}^{\nu} .
$$

Moreover, $M_{0}^{\nu}=X_{0}^{H, \nu}$. We obtain using Girsanoy theorem

$$
\begin{aligned}
d X_{t}^{H, \nu} & =\frac{1}{2 \gamma}\left(m_{t}^{2}+\nu_{t}^{2}\right) \mathrm{d} t+z_{t}^{1, \nu} \mathrm{d} \tilde{W}_{t}^{(1)}+z_{t}^{2, \nu} \mathrm{d} \tilde{W}_{t}^{(2)} \\
& =\frac{1}{2 \gamma}\left(m_{t}^{2}+\nu_{t}^{2}\right) \mathrm{d} t+z_{t}^{1, \nu} \mathrm{d} W_{t}^{(1)}+z_{t}^{2, \nu} \mathrm{d} W_{t}^{(2)}-z_{t}^{1, \nu} m_{t} \mathrm{~d} t-z_{t}^{2, \nu} \nu_{t} \mathrm{~d} t \\
& =\frac{1}{2 \gamma}\left[\left(m_{t}^{2}+\nu_{t}^{2}\right)-2 \gamma\left(z_{t}^{1, \nu} m_{t}+z_{t}^{2, \nu} \nu_{t}\right)\right] \mathrm{d} t+z_{t}^{1, \nu} \mathrm{d} W_{t}^{(1)}+z_{t}^{2, \nu} \mathrm{d} W_{t}^{(2)}
\end{aligned}
$$

We also remark that $X_{T}^{H, \nu}=H$.

Henceforth, $\left(X^{H, \nu}, z\right)$ verifies the backward stochastic differential equation ( BSDE in short)

$$
\left\{\begin{aligned}
-\mathrm{d} X_{t}^{H, \nu} & =f_{m, t}\left(z^{\nu}, \nu\right) \mathrm{d} t-z_{t}^{1, \nu} \mathrm{d} W_{t}^{(1)}-z_{t}^{2, \nu} \mathrm{d} W_{t}^{(2)}, \quad 0 \leq t \leq T \\
X_{T}^{H, \nu} & =H
\end{aligned}\right.
$$


with $f_{m, t}\left(z^{\nu}, \nu\right)=-\frac{1}{2 \gamma}\left[\left(m_{t}^{2}+\nu_{t}^{2}\right)-2 \gamma\left(z_{t}^{1, \nu} m_{t}+z_{t}^{2, \nu} \nu_{t}\right)\right], f_{m, t}\left(z^{\nu}, \nu\right)$ being the BSDE's generator.

The comparison's theorem suggests to characterize the process $X_{t}^{H}:=\operatorname{ess} \sup _{\nu} X_{t}^{H, \nu}$ as the solution of the BSDE of generator

$$
\hat{f}_{m, t}(z)=\max _{\nu} f_{m, t}(z, \nu)=f_{m, t}(z, \hat{\nu})
$$

with

and henceforth,

$$
\hat{\nu}=\gamma z^{(2)}
$$

$$
\hat{f}_{m, t}(z)=-\left[\frac{m_{t}^{2}}{2 \gamma}-m_{t} z_{t}^{(1)}-\frac{\gamma}{2}\left(z_{t}^{(2)}\right)^{2}\right] .
$$

Then we regard the BSDE with quadratic coefficients in $z$ :

$$
\left\{\begin{aligned}
-\mathrm{d} X_{t}^{H} & =\hat{f}_{m, t}(z) \mathrm{d} t-z_{t}^{(1)} \mathrm{d} W_{t}^{(1)}-z_{t}^{(2)} \mathrm{d} W_{t}^{(2)} \\
X_{T}^{H} & =H
\end{aligned}\right.
$$

An existence and uniqueness result of solution of BSDE with quadratic coefficients in [14], see also Section 1.3, allows to prove that the previous BSDE has an unique solution $\left(X^{H}, z\right)$,

with $\mathbb{E} \int_{0}^{T}\left(\left(z_{t}^{(1)}\right)^{2}+\left(z_{t}^{(2)}\right)^{2}\right) \mathrm{d} t<\infty$.

We deduce from comparison's theorem for quadratic BSDE (cf. Sect. 1.3) that:

$$
\forall \nu \in \mathcal{K}_{e}^{\prime} \quad X_{t}^{H} \geq X_{t}^{H, \nu} \text { a.s. }
$$

Lemma 3.1. We assume that the process $m_{t}$ is bounded.

Let $\hat{\nu}$ be the process defined by:

$$
\hat{\nu}_{t}=\gamma z_{t}^{(2)} \quad 0 \leq t \leq T,
$$

where $\left(X^{H}, z\right)$ is the unique solution of the previous BSDE.

We have $\hat{\nu} \in \mathcal{K}_{e}^{\prime}$ and $X_{t}^{H}=\mathbb{E}^{\mathbb{P}^{\hat{\nu}}}\left(H \mid \mathcal{F}_{t}\right)-\frac{1}{2 \gamma} \mathbb{E}^{\mathbb{P}^{\hat{\nu}}}\left(\int_{t}^{T}\left(m_{s}^{2}+\hat{\nu}_{s}^{2}\right) \mathrm{d} s \mid \mathcal{F}_{t}\right)$.

A consequence of this lemma is that $X_{t}^{H}=X_{t}^{H, \hat{\nu}}=\sup _{\nu \in \mathcal{K}_{e}{ }^{\prime}} \operatorname{ess} X_{t}^{H, \nu}$, and henceforth:

$$
X_{0}^{H}=\sup _{\mathbb{Q} \in \mathcal{M}_{e}^{\prime}}\left\{\mathbb{E}^{\mathbb{Q}}(H)-\frac{1}{\gamma} h(\mathbb{Q} \mid \mathbb{P})\right\}=\mathbb{E}^{\mathbb{P}^{\hat{\nu}}}\left(H-\frac{1}{2 \gamma} \int_{0}^{T}\left(m_{t}^{2}+\hat{\nu}_{t}^{2}\right) \mathrm{d} t\right) .
$$

Proof. We first prove that the process $X^{H}$ is bounded. We have:

$$
X_{t}^{H} \geq X_{t}^{H, 0}=\mathbb{E}^{\mathbb{P}^{0}}\left(H-\frac{1}{2 \gamma} \int_{t}^{T} m_{s}^{2} \mathrm{~d} s \mid \mathcal{F}_{t}\right)
$$

Since the process $\left(m_{t}\right)$ is bounded, we deduce that $X^{H}$ is uniformly lower bounded. We remark also that if we set:

$$
\tilde{f}_{m, t}(z)=m_{t} z^{(1)}+\frac{\gamma}{2}\left(z^{(2)}\right)^{2}
$$


the solution of the BSDE with generator $\tilde{f}_{m, t}$ and deterministic condition $C$ is given by

$$
\hat{X}_{t}=C \quad \hat{z}_{t}=0,0 \leq t \leq T \text {. }
$$

We have $\tilde{f}_{m, t}(z) \geq \hat{f}_{m, t}(z)$. The comparison's theorem for quadratic BSDE allows us to deduce that:

$$
X_{t}^{H} \leq \operatorname{supess} H, \quad 0 \leq t \leq T
$$

Let now $\left(\tau_{n}\right)$ be the sequence of stopping times defined by:

$$
\tau_{n}=\inf \left\{t \geq 0 \mid \gamma^{2} \int_{0}^{t}\left(z_{s}^{(2)}\right)^{2} \mathrm{~d} s \geq n\right\}
$$

The process $\nu^{(n)}$ defined by: $\nu_{t}^{(n)}=\gamma z_{t}^{(2)} I_{\left[0, \tau_{n}\right]}(t)$ is in $\mathcal{K}_{e}{ }^{\prime}$ and:

$$
X_{t \wedge \tau_{n}}^{H}=X_{0}^{H}+\frac{1}{2 \gamma} \int_{0}^{t \wedge \tau_{n}}\left(m_{s}^{2}+\left(\gamma z_{s}^{(2)}\right)^{2}\right) \mathrm{d} s+\int_{0}^{t \wedge \tau_{n}} z_{s}^{(1)} \mathrm{d} \tilde{W}_{s}^{(1)}+\int_{0}^{t \wedge \tau_{n}} z_{s}^{(2)} \mathrm{d} \tilde{W}_{s}^{(2)}
$$

with $\tilde{W}_{t}^{(1)}=W_{t}^{(1)}-\int_{0}^{t} m_{s} \mathrm{~d} s$ and $\tilde{W}_{t}^{(2)}=W_{t}^{(2)}-\int_{0}^{t} \gamma z_{s}^{(2)} \mathrm{d} s$.

Under $\mathbb{P}^{\nu^{(n)}}, \tilde{W}$ is a standard Brownian motion and we have:

$$
\mathbb{E}^{\mathbb{P}^{\nu(n)}} X_{t \wedge \tau_{n}}^{H}=X_{0}^{H}+\frac{1}{2 \gamma} \mathbb{E}^{\mathbb{P}^{\nu(n)}} \int_{0}^{t \wedge \tau_{n}}\left(m_{s}^{2}+\left(\gamma z_{s}^{(2)}\right)^{2}\right) \mathrm{d} s .
$$

If we note $M_{t}=\xi_{t}^{\hat{\nu}}$, then

$$
M_{t}=\exp \left(\int_{0}^{t}\left(m_{s} \mathrm{~d} W_{s}^{(1)}+\gamma z_{s}^{(2)} \mathrm{d} W_{s}^{(2)}\right)-\frac{1}{2} \int_{0}^{t}\left(m_{s}^{2}+\left(\gamma z_{s}^{(2)}\right)^{2}\right) \mathrm{d} s\right)
$$

and henceforth the previous equality can be written:

$$
\mathbb{E} M_{t \wedge \tau_{n}} \ln M_{t \wedge \tau_{n}}=\mathbb{E}^{\mathbb{P}^{\nu(n)}} X_{t \wedge \tau_{n}}^{H}-X_{0}^{H} .
$$

Since $X^{H}$ is bounded, we deduce that:

$$
\sup _{n} \mathbb{E} M_{t \wedge \tau_{n}} \ln M_{t \wedge \tau_{n}}<+\infty
$$

Hence the discrete parameter martingale $\left(M_{t \wedge \tau_{n}} \ln M_{t \wedge \tau_{n}}\right)_{n \in \mathbb{N}}$ is in the class $L \log L$ ([15], exercise 1.16, p. 58). We easily deduce that $M_{t}$ is a martingale and that $\mathbb{E} M_{t} \ln M_{t}<+\infty$ for $0 \leq t \leq T$; which proves that $\hat{\nu} \in \mathcal{K}_{e}{ }^{\prime}$. 


\section{Optimal trading Strategy}

This section is devoted to the proof of the theorem below.

Theorem 4.1. If $m_{t}$ is a bounded process, then the quantity defined in Section 1.2

$$
V(x, H)=-x+\sup _{\mathbb{Q} \in \mathcal{M}_{e}^{\prime}}\left\{\mathbb{E}^{\mathbb{Q}}(H)-\frac{1}{\gamma} h(\mathbb{Q} \mid \mathbb{P})\right\} .
$$

Furthermore, $V(x, H)=\frac{1}{\gamma} \ln \mathbb{E}\left[\exp \left(-\gamma\left(V_{T}^{x, \hat{\theta}}-H\right)\right)\right]$ with

$$
\hat{\theta}_{t}=-\frac{m_{t}}{\gamma \sigma_{t}^{g} S_{t}^{g}}+\frac{z_{t}^{(1)}}{\sigma_{t}^{g} S_{t}^{g}}
$$

where $\left(X^{H}, z\right)$ is the solution of $(3.2)$.

\subsection{Proof of the theorem 4.1}

Proof. We set $\hat{\mathbb{Q}}=\mathbb{P}^{\hat{\nu}}$. According to Lemma 3.1 we know that $\hat{\mathbb{Q}} \in \mathcal{M}_{e}{ }^{\prime}$.

We are seeking for $\hat{\theta} \in \mathcal{A}(x)$ such that

$$
\frac{1}{\gamma} \ln \mathbb{E}\left[\exp \left(-\gamma\left(V_{T}^{x, \hat{\theta}}-H\right)\right)\right]=-x+\mathbb{E}^{\hat{\mathbb{Q}}}(H)-\frac{1}{\gamma} h(\hat{\mathbb{Q}} \mid \mathbb{P}) .
$$

This equality is equivalent to

$$
\begin{aligned}
\ln \mathbb{E}\left[\exp \left(-\gamma\left(V_{T}^{x, \hat{\theta}}-H\right)\right)\right] & =-\gamma x+\mathbb{E}^{\hat{\mathbb{Q}}}(\gamma H)-h(\hat{\mathbb{Q}} \mid \mathbb{P}) \\
& =\mathbb{E}^{\hat{\mathbb{Q}}}(-\gamma(x-H))-h(\hat{\mathbb{Q}} \mid \mathbb{P}) \\
& =\mathbb{E}^{\hat{\mathbb{Q}}}\left(-\gamma\left(V_{T}^{x, \hat{\theta}}-H\right)\right)-h(\hat{\mathbb{Q}} \mid \mathbb{P})
\end{aligned}
$$

if $V^{x, \hat{\theta}}$ is a $\hat{\mathbb{Q}}$-martingale.

Hence according to the Lemma 2.1,

$$
\frac{\mathrm{d} \hat{\mathbb{Q}}}{\mathrm{dP}}=\frac{\exp \left(-\gamma\left(V_{T}^{x, \hat{\theta}}-H\right)\right)}{\mathbb{E} \exp \left(-\gamma\left(V_{T}^{x, \hat{\theta}}-H\right)\right)}
$$

We are led to search $\hat{\theta}$ such that $V^{x, \hat{\theta}}$ is a $\hat{\mathbb{Q}}$-martingale and

$$
\exp \left(-\gamma\left(V_{T}^{x, \hat{\theta}}-H\right)\right)=\mathrm{e}^{\gamma V(x, H)} \frac{\mathrm{d} \hat{\mathbb{Q}}}{\mathrm{d} \mathbb{P}}
$$

Hence

and

$$
-\gamma\left(V_{T}^{x, \hat{\theta}}-H\right)=\gamma V(x, H)+\ln \frac{\mathrm{d} \hat{\mathbb{Q}}}{\mathrm{d} \mathbb{P}}
$$

$$
V_{T}^{x, \hat{\theta}}=-V(x, H)+H-\frac{1}{\gamma} \ln \frac{\mathrm{d} \hat{\mathbb{Q}}}{\mathrm{d} \mathbb{P}} .
$$


As $V^{x, \hat{\theta}}$ must be a $\hat{\mathbb{Q}}$-martingale, we will in fact seek for $\hat{\theta}$ such that

$$
V_{t}^{x, \hat{\theta}}=-V(x, H)+\mathbb{E}^{\hat{\mathbb{Q}}}\left(H-\frac{1}{\gamma} \ln \frac{\mathrm{d} \hat{\mathbb{Q}}}{\mathrm{d} \mathbb{P}} \mid \mathcal{F}_{t}\right) .
$$

We know that

Furthermore,

$$
\begin{aligned}
X_{t}^{H}= & \operatorname{ess} \sup _{\mathbb{P}^{\nu} \in \mathcal{M}_{e}^{\prime}} \mathbb{E}^{\mathbb{P}^{\nu}}\left(H-\frac{1}{2 \gamma} \int_{t}^{T}\left(m_{s}^{2}+\nu_{s}^{2}\right) \mathrm{d} s \mid \mathcal{F}_{t}\right) \\
= & \mathbb{E}^{\hat{\mathbb{Q}}}\left(H-\frac{1}{2 \gamma} \int_{t}^{T}\left(m_{s}^{2}+\hat{\nu}_{s}^{2}\right) \mathrm{d} s \mid \mathcal{F}_{t}\right) .
\end{aligned}
$$

$$
\begin{aligned}
\mathbb{E}^{\hat{\mathbb{Q}}}\left(\ln \frac{\mathrm{d} \hat{\mathbb{Q}}}{\mathrm{d} \mathbb{P}} \mid \mathcal{F}_{t}\right)= & \mathbb{E}^{\hat{\mathbb{Q}}}\left(\int_{0}^{T} m_{t} \mathrm{~d} W_{t}^{(1)}+\int_{0}^{T} \hat{\nu}_{t} \mathrm{~d} W_{t}^{(2)}-\frac{1}{2} \int_{0}^{T}\left(m_{t}^{2}+\hat{\nu}_{t}^{2}\right) \mathrm{d} t \mid \mathcal{F}_{t}\right) \\
= & \int_{0}^{t} m_{s} \mathrm{~d} W_{s}^{(1)}+\int_{0}^{t} \hat{\nu}_{s} \mathrm{~d} W_{s}^{(2)}-\frac{1}{2} \int_{0}^{t}\left(m_{s}^{2}+\hat{\nu}_{s}^{2}\right) \mathrm{d} s \\
& +\mathbb{E}^{\hat{\mathbb{Q}}}\left(\int_{t}^{T} m_{s} \mathrm{~d} W_{s}^{(1)}+\int_{t}^{T} \hat{\nu}_{s} \mathrm{~d} W_{s}^{(2)}-\frac{1}{2} \int_{t}^{T}\left(m_{s}^{2}+\hat{\nu}_{s}^{2}\right) \mathrm{d} s \mid \mathcal{F}_{t}\right) \\
= & \ln \xi_{t}^{\hat{\nu}}+\mathbb{E}^{\hat{\mathbb{Q}}}\left(\int_{t}^{T} m_{s} \mathrm{~d} \tilde{W}_{s}^{(1)}+\int_{t}^{T} \hat{\nu}_{s} \mathrm{~d} \tilde{W}_{s}^{(2)}+\frac{1}{2} \int_{t}^{T}\left(m_{s}^{2}+\hat{\nu}_{s}^{2}\right) \mathrm{d} s \mid \mathcal{F}_{t}\right) \\
= & \ln \xi_{t}^{\hat{\nu}}+\mathbb{E}^{\hat{\mathbb{Q}}}\left(\frac{1}{2} \int_{t}^{T}\left(m_{s}^{2}+\hat{\nu}_{s}^{2}\right) \mathrm{d} s \mid \mathcal{F}_{t}\right) .
\end{aligned}
$$

Hence we have

$$
\begin{aligned}
V_{t}^{x, \hat{\theta}} & =-V(x, H)+\mathbb{E}^{\hat{\mathbb{Q}}}\left(H \mid \mathcal{F}_{t}\right)-\frac{1}{\gamma} \ln \xi_{t}^{\hat{\nu}}-\frac{1}{2 \gamma} \mathbb{E}^{\hat{\mathbb{Q}}}\left(\frac{1}{2} \int_{t}^{T}\left(m_{s}^{2}+\hat{\nu}_{s}^{2}\right) \mathrm{d} s \mid \mathcal{F}_{t}\right) \\
& =-V(x, H)-\frac{1}{\gamma} \ln \xi_{t}^{\hat{\nu}}+X_{t}^{H} .
\end{aligned}
$$

Then we find $\hat{\theta}$ : (for a better understanding we set $\hat{\nu}:=\tilde{\nu}$ )

$$
\begin{aligned}
-\frac{1}{\gamma} \mathrm{d} \ln \xi_{t}^{\tilde{\nu}} & =-\frac{1}{\gamma}\left[m_{t}\left(\mathrm{~d} \tilde{W}_{t}^{(1)}+m_{t} \mathrm{~d} t\right)-\frac{1}{2} m_{t}^{2} \mathrm{~d} t+\tilde{\nu}_{t}\left(\mathrm{~d} \tilde{W}_{t}^{(2)}+\tilde{\nu}_{t} \mathrm{~d} t\right)-\frac{1}{2} \tilde{\nu}_{t}^{2} \mathrm{~d} t\right] \\
& =-\left[\frac{m_{t}^{2}}{2 \gamma}+\frac{\gamma}{2}\left(z_{t}^{(2)}\right)^{2}\right] \mathrm{d} t-\frac{m_{t}}{\gamma} \mathrm{d} \tilde{W}_{t}^{(1)}-z_{t}^{(2)} \mathrm{d} \tilde{W}_{t}^{(2)} . \\
d X_{t}^{H} & =-\hat{f}_{m, t}(z) \mathrm{d} t+z_{t}^{(1)} \mathrm{d} \tilde{W}_{t}^{(1)}+z_{t}^{(2)} \mathrm{d} \tilde{W}_{t}^{(2)}+z_{t}^{(1)} m_{t} \mathrm{~d} t+z_{t}^{(2)} \tilde{\nu}_{t} \mathrm{~d} t \\
& =\left[-\hat{f}_{m, t}(z)+z_{t}^{(1)} m_{t} \mathrm{~d} t+z_{t}^{(2)} \tilde{\nu}_{t}\right] \mathrm{d} t+z_{t}^{(1)} \mathrm{d} \tilde{W}_{t}^{(1)}+z_{t}^{(2)} \mathrm{d} \tilde{W}_{t}^{(2)} \\
& =\left[-\hat{f}_{m, t}(z)+m_{t} z_{t}^{(1)}+\gamma\left(z_{t}^{(2)}\right)^{2}\right] \mathrm{d} t+z_{t}^{(1)} \mathrm{d} \tilde{W}_{t}^{(1)}+z_{t}^{(2)} \mathrm{d} \tilde{W}_{t}^{(2)} \\
& =\left[\frac{m_{t}^{2}}{2 \gamma}+\frac{\gamma}{2}\left(z_{t}^{(2)}\right)^{2}\right] \mathrm{d} t+z_{t}^{(1)} \mathrm{d} \tilde{W}_{t}^{(1)}+z_{t}^{(2)} \mathrm{d} \tilde{W}_{t}^{(2)} .
\end{aligned}
$$


We get

$$
\begin{aligned}
\mathrm{d} V_{t}^{x, \hat{\theta}}= & -\frac{1}{\gamma} \mathrm{d} \ln \xi_{t}^{\hat{\nu}}+\mathrm{d} X_{t}^{H} \\
= & \left(\frac{-m_{t}}{\gamma}+z_{t}^{(1)}\right) \mathrm{d} \tilde{W}_{t}^{(1)} \\
= & \left(\frac{-m_{t}}{\gamma}+z_{t}^{(1)}\right) \frac{\sigma_{t}^{g} S_{t}^{g}}{\sigma_{t}^{g} S_{t}^{g}} d \tilde{W}_{t}^{(1)} \\
& \frac{-m_{t}}{\gamma}+z_{t}^{(1)} \\
= & \mathrm{d} S_{t}^{g} \cdot \\
\hat{\theta}_{t} & =-\frac{m_{t} S_{t}^{g}}{\gamma \sigma_{t}^{g} S_{t}^{g}}+\frac{z_{t}^{(1)}}{\sigma_{t}^{g} S_{t}^{g}} .
\end{aligned}
$$

It remains to show that $\hat{\theta} \in \mathcal{A}(x)$ and that $V^{x, \hat{\theta}}$ is a $\hat{\mathbb{Q}}$-martingale. Finally we will have that $\hat{\theta}$ is the optimal trading strategy.

We recall that $X_{t}^{H}=\mathbb{E}^{\mathbb{P}^{\hat{\nu}}}\left(H-\frac{1}{2 \gamma} \int_{t}^{T}\left(m_{s}^{2}+\hat{\nu}_{s}^{2}\right) \mathrm{d} s \mid \mathcal{F}_{t}\right)$.

We define the sequence of trading strategies

$$
\begin{gathered}
\theta_{n}=\hat{\theta} 1_{\left[0, \tau_{n}\right]} \text { with } \tau_{n}=\inf \left\{t \in[0, T] \mid V_{t}^{x, \hat{\theta}} \leq-n\right\} \\
\theta_{n} \in \mathcal{A}_{b}(x) \text { since } V_{t}^{x, \theta_{n}}=V_{t \wedge \tau_{n}}^{x, \hat{\theta}} \geq-n
\end{gathered}
$$

We have

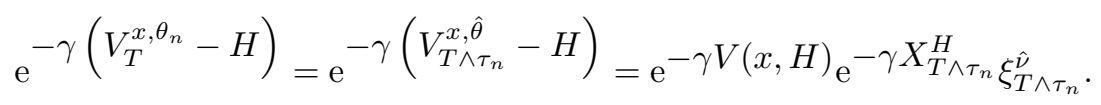

We want to show that $\mathrm{e}^{-\gamma\left(V_{T}^{x, \theta_{n}}-H\right)}$ converges in $L^{1}(\mathbb{P})$ towards $\mathrm{e}^{-\gamma\left(V_{T}^{x, \hat{\theta}}-H\right)}(\hat{\theta} \in \mathcal{A}(x))$.

As $\xi^{\hat{\nu}}$ is a martingale, the sequence $\left(\xi_{T \wedge \tau_{n}}^{\hat{\nu}}\right)_{n \geq 1}$ is uniformly integrable, and since $X^{H}$ is bounded, so does $e^{-\gamma\left(V_{T}^{x, \theta_{n}}-H\right)}$. Almost sure convergence and uniform integrability imply the convergence in $L^{1}(\mathbb{P})$.

We deduce that $\hat{\theta} \in \mathcal{A}(x)$.

Remark 4.1. The process $m_{t}$ is bounded. The equality $V(x+p, H)=V(x, 0)$ implies that the indifference price $(c f .[8])$ is

$$
p=\sup _{\mathbb{Q} \in \mathcal{M}_{e}}\left\{\mathbb{E}^{\mathbb{Q}}(H)-\frac{1}{\gamma} h(\mathbb{Q} \mid \mathbb{P})\right\}-\sup _{\mathbb{Q} \in \mathcal{M}_{e}}\left\{-\frac{1}{\gamma} h(\mathbb{Q} \mid \mathbb{P})\right\}
$$




\subsection{The case $\rho=1$}

In this case we get

$$
\frac{\mathrm{d} S_{s}^{e}}{S_{s}^{e}}=\left(\mu_{s}^{e}+\sigma_{s}^{e} m_{s}\right) \mathrm{d} s+\sigma_{s}^{e} \rho_{s} \mathrm{~d} \tilde{W}_{s}^{(1)}
$$

i.e. there is only one martingale measure denoted previously $\mathbb{P}^{0}:=\left.\mathbb{P}^{\nu}\right|_{\nu \equiv 0}$ and it is clear that

$$
X_{.}^{H}=\mathbb{E}^{\mathbb{P}^{0}}\left(H-\frac{1}{2 \gamma} \int^{T} m_{s}^{2} \mathrm{~d} s \mid \mathcal{F} .\right) .
$$

To study then the indifference price one has to deal with no arbitrage argument. Indeed when $\rho=1$, the two assets of spot prices $S^{e}$ and $S^{g}$ are perfectly correlated. Then for instance if we assume that the coefficients of the model are constant and $\sigma^{g}$ strictly positive we have the relation, see [4],

$$
\mu^{e}=\mu^{g} \frac{\sigma^{e}}{\sigma^{g}}
$$

between the coefficients, to avoid arbitrage opportunities.

If we denote by $p_{t}^{H}$ the indifference price process defined by

$$
p^{H}=X^{H}-X^{0}
$$

then it is clear that $p_{t}^{H}=\mathbb{E}^{\mathbb{P}^{0}}\left(H \mid \mathcal{F}_{t}\right)$ which is the Black-Scholes price. Using the BSDE techniques we have developped in the preceding sections, if we set

$$
z^{H}=z^{(1)}-\tilde{z}^{(1)}
$$

with $\left(X^{H}, z\right)$ and $\left(X^{0}, \tilde{z}\right)$ being solutions for the BSDE (3.2), then we have

$$
-\mathrm{d} p_{t}^{H}=m_{t} z_{t}^{H} \mathrm{~d} t-z_{t}^{H} d W_{t}^{(1)}, p_{T}^{H}=H
$$

i.e.

$$
\begin{aligned}
-\mathrm{d} p_{t}^{H} & =-z_{t}^{H}\left(\mathrm{~d} W_{t}^{(1)}-m_{t} \mathrm{~d} t\right) \\
& =-z_{t}^{H} \mathrm{~d} \tilde{W}_{t}^{(1)} .
\end{aligned}
$$

Hence, also, we obtain

$$
p_{t}^{H}=\mathbb{E}^{\mathbb{P}^{0}}\left(H \mid \mathcal{F}_{t}\right) .
$$

Henceforth, when $m_{t}$ is bounded, the exponential criterion can be viewed as an extension of Black-Scholes style pricing.

\section{Conclusion}

We characterize the optimal solution to the program of maximizing exponential utility from net terminal wealth for an agent, in the situation of incomplete markets. This is done, by an appropriate definition of the domain of validity of the dual problem. Nevertheless, we were forced to limit ourselves to the case of a bounded 
risk premium process, opening thus our work to further research in view of the relaxation of such a condition. Another way of continuing the research will be to study in our general model the expansion of the hedging near the perfect correlation case, by the bias of Malliavin calculus. Our study can be applied to the hedging of temperature weather derivatives and of index options. We have fully solved the dual problem by means, which to our knowledge, are original. In addition, we have got out Lemma 3.1 which allows us to emphasize the assumption that $m_{t}$ is bounded to well determine the optimal hedging strategy (cf. Th. 4.1).

\section{APPENDIX}

Now we state the following decomposition theorem of local martingales (Th. 1.1):

Proof. We first regard the case $N \overline{\mathbb{Q}}$-martingale. Then, following [13], Proposition 8.6 p. 375, we have such a decomposition of $N$. We will only prove that if $N_{T} \in L^{2}(\overline{\mathbb{Q}})$, then $\mathbb{E}^{\overline{\mathbb{Q}}}<N, N>_{T}<+\infty$.

Let $\tau_{n}=\inf \left\{t \in[0, T] \mid\langle N, N\rangle_{t} \geq n\right.$ and $\left.N_{t} \geq n\right\}, n \geq 1$.

The increasing sequence of stopping times $\left(\tau_{n}\right)_{n \geq 1}$ converges towards $+\infty$;

$\left(N_{t \wedge \tau_{n}}\right)_{0 \leq t \leq T}$ is a square integrable $\overline{\mathbb{Q}}$-martingale, for any $n \geq 1$ :

$\mathbb{E}^{\overline{\mathbb{Q}}}\left(N_{T \wedge \tau_{n}}^{2}\right)=\mathbb{E}^{\overline{\mathbb{Q}}}\langle N, N\rangle_{T \wedge \tau_{n}}=n<+\infty$. By continuity of $t \longmapsto N_{t}$ and by using the monotone convergence theorem, we have:

$$
\begin{aligned}
\mathbb{E}^{\overline{\mathbb{Q}}}\left(N_{T}^{2}\right) & =\mathbb{E}^{\overline{\mathbb{Q}}} \lim _{n} N_{T \wedge \tau_{n}}^{2} \\
& =\lim _{n} \mathbb{E}^{\overline{\mathbb{Q}}}\left(N_{T \wedge \tau_{n}}^{2}\right) \\
& =\mathbb{E}^{\overline{\mathbb{Q}}}\langle N, N\rangle_{T}<+\infty
\end{aligned}
$$

The end of the proof is done in the proof of the Theorem 3.4, Ch. V, p. 200, [15].

\section{REFERENCES}

[1] D. Becherer, Rational Hedging and Valuation with Utility-Based Preference. PhD Thesis, Berlin University (2001).

[2] D. Becherer, Rational Hedging and Valuation of Integrated Risks under Constant Absolute Risk Aversion, Insurance: Math. Econ. 33 (2003) 1-28.

[3] J. Cvitanic, W. Schachermayer and H. Wang, Utility Maximization in Incomplete Market with Random Endowment, in Proceedings of Symposia in Applied Mathematics (1999).

[4] M. Davis, Optimal Hedging with Basis Risk, preprint (2000).

[5] M. Davis, Option Valuation and Hedging with Basis Risk, in System Theory: Modeling, Analysis and Control, T.E. Djaferis and I.C. Schick Eds., Kluwer, Amsterdam (1999).

[6] F. Delbaen and W. Schachermayer, Arbitrage and Free Lunch with Bounded Risk for Unbounded Continuous Processes, Mathematical Finance 4 (1994) 343-348.

[7] F. Delbaen, P. Grandits, T. Rheinlander, D. Samperi, M. Schweizer and C. Stricker, Exponential Hedging and Entropic Penalties. Mathematical Finance 12 (2002) 99-123.

[8] N. El Karoui and R. Rouge, Pricing via Utility Maximization and Entropy, Mathematical Finance 10 (2000) 259-276.

[9] W.H. Fleming and R.W. Rishel, Deterministic and Stochastic Optimal Control. Springer Verlag, New York (1975).

[10] V. Henderson, Valuation of Claims of Non Traded Assets using Utility Maximization, Mathematical Finance 12 (2002) 351-373.

[11] Y.M. Kabanov and C. Stricker, On the Optimal Portfolio for the Exponential Utility Maximization: Remarks to the Six-Author Paper, Mathematical Finance 12 (2002) 125-134.

[12] I. Karatzas and S.E. Shreve, Methods of Mathematical Finance. Springer Verlag, New York (1998).

[13] I. Karatzas and S.E. Shreve, Brownian Motion and Stochastic Calculus. Springer Verlag (1991). 
[14] M. Kobylanski, Backward Stochastic Differential Equations and Partial Differential Equations with Quadratic Growth, The Annals of Probability 2 (2000) 558-602.

[15] D. Revuz and M. Yor, Continuous Martingales and Brownian Motion. Springer Verlag (1991).

[16] W. Schachermayer, Optimal Investment in an Incomplete Market, in H. Geman et al. Eds. Mathematical Finance Bachelier Congress (2000), Berlin Heidelberg New York, Springer (2002).

[17] M. Yor. Sous-Espaces Denses dans $L^{1}$ ou $H^{1}$, in Séminaire de Probabilités XII, Springer Verlag (1978) 265-309. 